Original Research

\title{
Ginsenoside Rb1 attenuates lipopolysaccharide-induced neural damage in the brain of mice via regulating the dysfunction of microglia and astrocytes
}

\author{
Huiming Zhang ${ }^{1}$, Xue Chen $^{1}$, Xi Wang $^{1}$, Ying Liu ${ }^{1}$, Charles. D. Sands ${ }^{2}$, Minke Tang $^{1, *}$ \\ ${ }^{1}$ School of Chinese Materia Medica, Beijing University of Chinese Medicine, 100029 Beijing, China \\ ${ }^{2}$ McWhorter, School of Pharmacy, College of Health Sciences, Samford University, Birmingham, AL 35229, USA \\ *Correspondence: tangmk@bucm.edu.cn (Minke Tang) \\ DOI:10.31083/j.jin2004084 \\ This is an open access article under the CC BY 4.0 license (https://creativecommons.org/licenses/by/4.0/). \\ Submitted: 3 June 2021 Revised: 16 July 2021 Accepted: 23 July 2021 Published: 30 December 2021
}

The purpose of our research was to evaluate whether ginsenoside $\mathrm{Rb} 1$ has neuroprotective effects against lipopolysaccharide (LPS)induced brain injury. ICR mice were intraperitoneally (i.p.) injected with 20 or $40 \mathrm{mg} / \mathrm{kg} \mathrm{Rb} 1$ or saline for 7 consecutive days. On the 7th day, 30 minutes after Rb1 or saline administration, a single dose of LPS (LPS group, Rb1+LPS group) or saline (control group) was injected i.p. into the mice. Results demonstrated that Rb1 treatment could significantly improve the behavior performance of LPS mice in both the open field test and the beam walking test. Rb1 can also markedly attenuate the neuronal lesion in both hippocampus and somatosensory cortex in the brain of LPS mice. In addition, Rb1 treatment also significantly inhibits the LPS-induced neuroinflammation in the brain, indicated by reduced reactive microglia and decreased $\mathrm{IL}-1 \beta$ production. Both immunostaining and western blot results suggest that Rb1 can further enhance the LPS-induced CLT-1 expression and alleviate LPS-induced CS reduction in the brain. Our findings show that $\mathrm{Rb} 1$ has a protective effect on LPS-induced neuronal damage in the CA1 of the hippocampus and in the somatosensory area of the cerebral cortex in mice, which is likely to be the basis for its improvement of locomotor and motor coordination. Rb1 regulating the function of astrocytes and microglia through CLT-1 and CS in astrocytes may be involved in its neuroprotective effects.

\section{Keywords}

Cinsenoside Rb1; LPS-induced mice; Locomotive activity; Microglia; Astrocyte; Glutamate transporter-1; Clutamine synthetase enzyme

\section{Introduction}

Neuroinflammation is a well-documented pathological event in neurodegenerative diseases, such as Alzheimer's disease, Parkinson's disease, and amyotrophic lateral sclerosis [1, 2]. It is considered an important contributor to the pathogenesis and progression of neurodegeneration [1]. Lipopolysaccharide (LPS), a gram-negative endotoxin, is well-known to mimic inflammatory conditions when administered to animals. The systemic inflammation by a single exposure to LPS transfers to the brain and primes a vicious circle of neuroinflammation with disruption of the blood-brain barrier (BBB), activation of glial cells, and production of a vari- ety of proinflammatory cytokines in the brain that is detrimental to neurons [3-5]. Consequent cognitive impairment and depression-like behavior are also well documented [6-8]. Therefore, LPS-treated animals are widely used as neuroinflammation models of neuroprotection against neurodegeneration.

Glial cells, such as astrocytes, microglia, and oligodendrocytes, constitute a large fraction of the mammalian brain. Originally considered non-functional support for neurons, more and more research has highlighted the importance of glial cells in normal brain function [1,2]. Astrocytes perform several necessary functions for the formation and maintenance of the $\mathrm{BBB}$ and regulating brain water and ion homeostasis [9]. One of the major functions of astrocytes within the CNS is to uptake excitatory glutamate, which is of central importance for maintaining glutamate-glutamine homeostasis to prevent neuron damage from glutamate-mediated excitotoxicity [10]. For microglial cells, continuous and rapid surveillance is their unique feature in the brain. Microglia cells can recognize and engulf injured cells to prevent expanded neuron damage $[2,11]$. However, excessive activation of microglia cells is deleterious through self-propelling neuroinflammation, which impairs the optimal milieu for neuronal function, thereby contributing to neurodegeneration $[2,11]$.

Ginseng has been used worldwide as traditional medicine for thousands of years. Several ingredients with multiple activities have been isolated from Ginseng, such as $\operatorname{Rg} 1, \mathrm{Rb} 1$, Rh2, Rg3, Rg5, and Re. Particularly, Rb1 has been proven more effective among all other ginsenosides in terms of antiinflammation and repairing neuronal cell loss $[12,13]$. We previously found that Rb1 has significant effects in enhancing the expression of GLT-1 in astrocytes under oxygen-glucose deprivation and reoxygenation conditions (unpublished results). Given the importance of GLT-1 in regulating glial glutamate uptake and its consequent role in neuroinflammation [14], it is of value to investigate the effect of Rb1 on LPS-treated neuroinflammation and related neuronal le- 


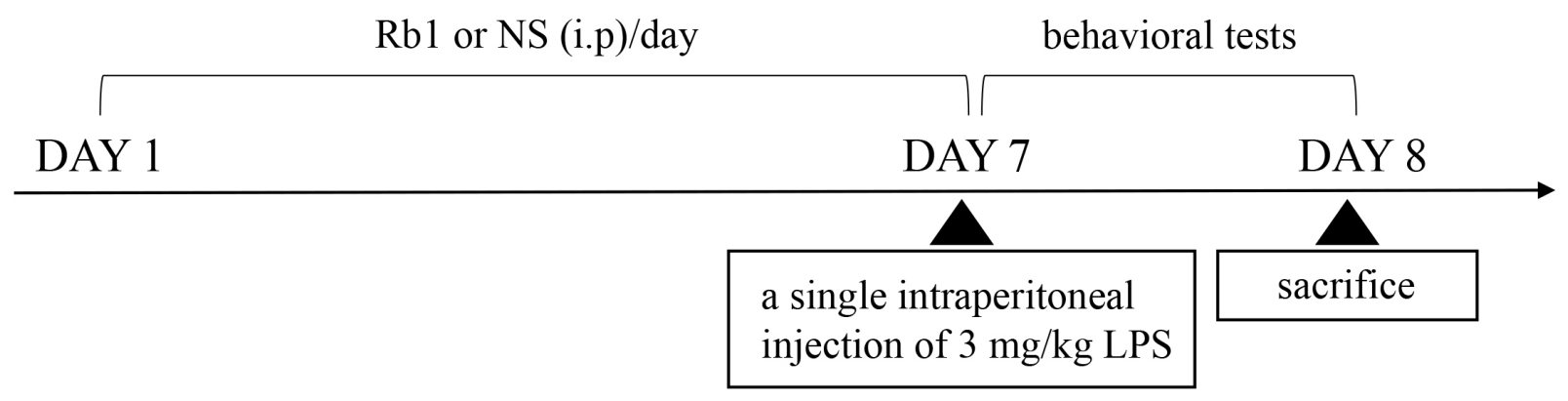

Fig. 1. The experimental design. ICR mice were intraperitoneally (i.p.) injected with 20 or $40 \mathrm{mg} / \mathrm{kg}$ Rb1 or saline for 7 consecutive days. On the 7 th day, 30 minutes after Rb1 or saline administration, a single dose of LPS (LPS group, Rb1+LPS group) or saline (control group) was injected i.p. into the mice. The locomotive activity was evaluated using the open-field test and the beam walking test at 20-24 h after the LPS injection. Mice were anesthetized with $7 \mathrm{~mL} / \mathrm{kg}$ $1 \%$ pentobarbital sodium and transcardially perfused with ice-cold NS and 4\% PFA $24 \mathrm{~h}$ after LPS injection for subsequent immunohistochemistry. Mice were anesthetized with $7 \mathrm{~mL} / \mathrm{kg} 1 \%$ pentobarbital sodium and sacrificed $24 \mathrm{~h}$ after LPS injection for subsequent Western blot analysis and ELISA.

sions. We used LPS to induce neuroinflammation in mice and investigate the effects of Rb1. The results show that the administration of $\mathrm{Rb} 1$ in advance can significantly reduce the impairment of locomotor function and motor coordination caused by LPS. Rb1 significantly reduces neuroinflammation and neuronal cell damage both in the hippocampus and cerebral cortex. In addition, Rb1 can further enhance the LPSinduced GLT-1 expression and alleviate LPS-induced GS reduction in the brain.

\section{Materials and methods \\ 2.1 Animals}

Six-week-old male ICR mice (29-31 g, n = 80) were purchased from Vital River Laboratory Animal Technology Co., Ltd. in Beijing, China (certificate number, SCXK [Jing] 2016-0006). Mice in all groups were kept at $23 \pm 1{ }^{\circ} \mathrm{C}$ and a 12-h light/dark cycle (light on 7:00-19:00). During the experimental period, the mice were given free access to tap water and food. All animal experiments were carried out in accordance with the National Institutes of Health Guide for Care and Use of Laboratory Animals [15], and the Institutional Animal Care and Use Committee of Beijing University of Chinese Medicine approved the protocol (BUCM-42020100803-4136, 8 October 2020). All efforts were made to minimize animal suffering and reduce the number of animals used.

\subsection{Group and treatment}

After 3 days of mice were fed, mice $(n=80)$ were randomly assigned to four groups ( $\mathrm{n}=20$ per group), i.e., the control group, the LPS-treated group (LPS group), the 20 $\mathrm{mg} / \mathrm{kg}$ Rb1 group (Rb1 20+LPS group) and the $40 \mathrm{mg} / \mathrm{kg}$ $\mathrm{Rb} 1$ group (Rb1 40+LPS group).

Rb1 (purity >98\%, Chengdu Must Bio-Technology, Chengdu, China) was dissolved in normal saline (NS) (Shijiazhuang No. 4 Pharmaceutical, Shijiazhuang, China). Mice were administered $\mathrm{Rb} 1$ intraperitoneally (i.p.) to achieve $20 \mathrm{mg} / \mathrm{kg} /$ day (Rb1 20+LPS group) or $40 \mathrm{mg} / \mathrm{kg} /$ day (Rb1 40+LPS group) for a week (9:00-11:00 AM) (Fig. 1). The control and LPS-treated groups were treated with NS. To create a systemic inflammatory model, a single intraperitoneal injection of $3 \mathrm{mg} / \mathrm{kg}$ LPS (Escherichia coli, 0111: B4, Sigma, St. Louis, MO, USA) dissolved in NS was given to each mouse within 15-30 min after the last Rb1 injection or NS injection (excluding the control group).

\subsection{Open-field test}

The locomotive activity was evaluated using the openfield test at $20 \mathrm{~h}$ after the LPS injection (Fig. 1). Each mouse (n $=20$ per group) was placed in the center of an open field apparatus (W50 $\times \mathrm{D} 50 \times \mathrm{H} 40 \mathrm{~cm}$ ), and free moving behavior was monitored for 5 minutes, and their behavior was recorded by the animal behavior analysis system (Etho-Vision XT9, Noldus, Wageningen, The Netherlands). The total distance moved and the crossing lines, moving and not moving durations, and velocity was analyzed. The open field apparatus was cleaned with 70\% ethanol (Sinopharm Chemical Reagent Co., Ltd, Shanghai, China), and it was ensured that ethanol evaporated fully between trials.

\subsection{Beam walking test}

Mice ( $\mathrm{n}=20$ per group) were trained for two days to walk from a start platform along a square beam $(80 \mathrm{~cm}$ long, $3 \mathrm{~cm}$ wide) elevated $30 \mathrm{~cm}$ above the bench by supports to a goal box. Three trials to traverse the beam were performed for each mouse daily, and mice were excluded from the cohort if they could still not traverse the beam autonomously and smoothly after 2 days of training. On the third day, the mice were tested on the cylindrical beam $(80 \mathrm{~cm}$ long, $8 \mathrm{~mm}$ diameter) after the open field test. The time to traverse each beam (up to $60 \mathrm{~s}$ ) was recorded, and the performance on the beam was evaluated by the scoring system adapted from the method of Feeney et al. [16-18]. The higher the score, the better the mice performed. Mice that traversed the beam normally with no foot slip were given a score of " 5 "; those that traversed the beam successfully and foot slips occurred on less than half of the way were given a score of " 4 "; those that traversed the beam successfully and foot slips occurred 
on more than half of the way were given a score of " 3 ". Mice that fell from the beam were given a score of " 2 ", and those unable to place limbs on the beam surface were given a score of " 1 ". Such situations were allotted a maximum latency of 60 seconds. The walking beam apparatus was cleaned with $70 \%$ ethanol, and it was ensured that ethanol evaporated fully between trials.

\subsection{Double immunohistochemistry}

Mice ( $\mathrm{n}=6$ per group) were anesthetized with $7 \mathrm{~mL} / \mathrm{kg} 1 \%$ pentobarbital sodium (Sigma, St. Louis, MO, USA) and transcardially perfused with ice-cold NS and 4\% paraformaldehyde (PFA) (Servicebio, Wuhan, China) $24 \mathrm{~h}$ after LPS injection. The brains were then placed in PFA and were preserved by paraffin embedding. Sagittal sections at $5 \mu \mathrm{m}$ were deparaffinized and were placed in a repair box filled with EDTA antigen retrieval buffer ( $\mathrm{pH}$ 8.0) (Servicebio, Wuhan, China) in a microwave oven for antigen retrieval. Non-specific proteins were blocked using 5\% BSA (Servicebio, Wuhan, China) in PBS (Servicebio, Wuhan, China) for 30 minutes before incubation in a cocktail of primary antibodies, i.e., rabbit polyclonal anti-GLT-1 (1:1000; Proteintech, Rosemont, IL, USA)+mouse polyclonal anti-GFAP (1:800; Servicebio, Wuhan, China), rabbit monoclonal antiGS (1:1000; Proteintech, Rosemont, IL, USA)+mouse polyclonal anti-GFAP (1:800; Servicebio, Wuhan, China) and mouse polyclonal anti-NeuN (1:200; Servicebio, Wuhan, China)+rabbit monoclonal anti-IBa-1 (1:200; Servicebio, Wuhan, China), overnight at $4{ }^{\circ} \mathrm{C}$. The sections were washed three times for $5 \mathrm{~min}$ in PBS ( $\mathrm{pH}$ 7.4) (Servicebio, Wuhan, China) and further incubated with an appropriate combination of fluorochrome-tagged secondary antibodies, i.e., Cy3labeled Goat Anti-Rabbit IgG (Servicebio, Wuhan, China), Alexa Fluor ${ }^{\circledR}$ 488-labeled Goat Anti-Mouse IgG (Servicebio, Wuhan, China), at a dilution of 1:500 for $50 \mathrm{~min}$ at room temperature. After proper washing in PBS were incubated with DAPI (Servicebio, Wuhan, China) for $10 \mathrm{~min}$ at room temperature in the dark and were mounted with an antifade mounting medium after PBS washing. Images of the brain were scanned with the Pannoramic Digital Slide Scanners (Pannoramic MIDI, 3D HISTECH, Budapest, Hungary) and were captured with Caseviewer 2.3 (3DHISTECH Ltd, Budapest, Hungary). The quantitative analysis of immunostained cells per field was done using Image J software (National Institutes of Health, Bethesda, MD, USA). Five visual fields were randomly selected, and inconsistency in background intensity was abolished. The pyramidal cells of the hippocampus appeared in a multilayered and compact arrangement, so the neurons in the CA1 stained by immunofluorescence were of slightly lower resolution. When counting the number of neurons in the CA1 region of the hippocampus, it was more accurate to count the number of double positive cells that DAPI co-label with NeuN on the CA1 using Image $\mathrm{J}$ software.

\subsection{Western blot analysis}

Mice ( $\mathrm{n}=3$ per group) were anesthetized with $7 \mathrm{~mL} / \mathrm{kg} 1 \%$ pentobarbital sodium and sacrificed $24 \mathrm{~h}$ after LPS injection. The brains were collected and deep-frozen in liquid nitrogen followed by transfer to $-80{ }^{\circ} \mathrm{C}$ for further analysis. The brain tissue was homogenized and sonicated on ice in RIPA lysis buffer (KeyGen Biotech, Nanjing, China) and protease inhibitor cocktail (KeyGen Biotech, Nanjing, China). After centrifugation, the supernatant was collected and assayed for protein concentration using the BCA protein quantitation assay (KeyGen Biotech, Nanjing, China). Lysate samples containing $10 \mu \mathrm{g}$ of protein were fractionated by $10 \%$ SDS-PAGE for $45 \mathrm{~min}$ at $60 \mathrm{~V}$ and then $75 \mathrm{~min}$ at $100 \mathrm{~V}$, and transferred onto a polyvinylidene difluoride (PVDF) membrane (Millipore, Boston, MA, USA) under ice bath conditions about 65 min $100 \mathrm{~V}$. The PVDF membrane was blocked in newly prepared 5\% novisualizedn-fat powdered milk (BioRuler, Danbury, CT, USA) for $1 \mathrm{~h}$ at room temperature and incubated with primary antibodies for GLT-1 (1:1000; rabbit monoclonal antibody, Abcam, Cambridge, MA, USA), GS (1:4000; rabbit polyclonal antibody, Proteintech, Rosemont, IL, USA), $\beta$-actin (1:12000; mouse monoclonal antibody, Proteintech, Rosemont, IL, USA), and $\beta$-tubulin (1:8000; rabbit polyclonal antibody, Proteintech, Rosemont, IL, USA) overnight at $4{ }^{\circ} \mathrm{C}$. Membranes were washed with $1 \%$ TBST (Servicebio, Wuhan, China) three times for $5 \mathrm{~min}$ each and incubated with HRP-conjugated affinipure goat anti-mouse or rabbit IgG (1:5000; Proteintech, Rosemont, IL, USA) for $1 \mathrm{~h}$ at room temperature. Blots were detected with enhanced chemiluminescence reagent (Analysis Quiz, Beijing, China) and the automatic chemiluminescence image system (Bio-Rad, Hercules, CA, USA). The density of the target protein and $\beta$-actin was measured using Image $\mathrm{J}$ software.

\subsection{ELISA}

Mice ( $\mathrm{n}=8-11$ per group) were anesthetized with 7 $\mathrm{mL} / \mathrm{kg} 1 \%$ pentobarbital sodium (Sigma, St. Louis, MO, USA) and sacrificed $24 \mathrm{~h}$ after LPS injection. The brains were collected and deep-frozen in liquid nitrogen and stored at $80^{\circ} \mathrm{C}$ until subsequent ELISA. Tissues were weighed and homogenized in ice-cold normal saline, and amounts of IL- $1 \beta$ in the mouse brain were determined with ELISA Kits (Raybiotech, Norcross, GA, USA) according to the manufacturer's instructions. The protein concentrations were measured using a BCA protein assay (KeyGen Biotech, Nanjing, China), and their ratio was used as the relative value of IL- $1 \beta$.

\subsection{Statistical analysis}

For statistical analysis, all data are presented using GraphPad Prism 6.01 (GraphPad Software Inc., San Diego, CA, USA) for statistical analysis. One-way analysis of variance (ANOVA) followed by an LSD test or Tamhane's T2 test was performed. And if the data were not normal, Kruskal-Wallis of the non-parametric test was performed; $p$ values of $p<$ 0.05 were considered significant. All data are shown as mean 
\pm SD. Statistical analyses were performed using SAS 9.4 software (SAS Inc., Raleigh, NC, USA) and SPSS Statistics 25.0 (IBM, Armonk, NY, USA).

\section{Results}

3.1 Rb1 improves the locomotive activity in the open-field test following the LPS challenge

It has been reported that systemic inflammation induced by LPS can lead to behavioral disorders, including sensorimotor deficits and cognitive impairment. Thus, we firstly investigated the effects of Rb1 on the LPS-induced behavior changes by using the open-field test, which is regarded as a comprehensive quantitative assessment of voluntary activities and exploration behaviors [19]. Compared to control mice, reduced voluntary locomotor activity as indicated by slow walking and decreased activity was observed in mice treated with LPS, as shown in Fig. 2. Rb1 treatment improved the abnormal behavior caused by LPS, manifested as prolonged movement distance and increased movement speed (Fig. 2B-F), although the dose-effect relationship is not obvious. The results conclusively indicate that $\mathrm{Rb} 1$ relieves the behavior changes caused by LPS and improves locomotive activity.

\subsection{Rb1 improves motor coordination deficits induced by LPS}

It has been reported that the beam walking assay is a more sensitive tool for monitoring sensorimotor function in rodents than locomotor studies [16]. To further examine the effect of Rb1 on motor impairment in LPS mice, we investigated motor coordination using the beam walking test with some modification $[16,18,20]$. The crossing performance is scored, and the time crossing the beam is recorded. A higher score indicates a better crossing performance. After two days of training, most mice can cross the beam within a few seconds. Mice with poor training performance were excluded from the cohort. On the third day, the beam walking performance was tested. There were no foot slips or falls observed in control mice (Fig. 3A). However, as shown in Fig. 3, LPS mice were becoming slow when crossing the beam, and a significant number of foot slips or falls were observed in LPStreated mice (Fig. 3B,D). Some of the LPS-treated mice could not cross the beam, and some could not even place their limbs on the beam (Fig. 3C). Compared to LPS mice, the mice in the $20 \mathrm{mg} / \mathrm{kg}$ and $40 \mathrm{mg} / \mathrm{kg}$ groups had a much better performance crossing the beam, with fewer foot slips (Fig. 3E,F). No falls were observed in both the Rb1 $20 \mathrm{mg} / \mathrm{kg}$ and 40 $\mathrm{mg} / \mathrm{kg}$ groups. The results suggest that $\mathrm{Rb} 1$ improved the motor coordination of LPS treated mice.

\subsection{Rb1 attenuates LPS-induced neuron injury in the brain}

The primary somatosensory cortex and hippocampus are important sections of the brain responsible for regulating voluntary and involuntary movement. They also facilitate the control of motor coordination [21]. To explore the underlying reason for the locomotor changes, we investigated the neuronal injury in the cortex and hippocampus by immunostaining neurons with $\mathrm{NeuN}$, a neuronal nuclear marker [22], in S1 Tr, S1BF, and S2 areas of the somatosensory cortex and CA1 in the hippocampus (Fig. 4A). As shown in Fig. 4, LPS treatment mildly reduced the NeuN in all the above regions compared to the control mice (Fig. 4B-D). $\mathrm{Rb} 120 \mathrm{mg} / \mathrm{kg}$ and $40 \mathrm{mg} / \mathrm{kg}$ treatments significantly attenuated LPS-induced neuronal loss (Fig. 4B-D). However, $\mathrm{Rb} 140 \mathrm{mg} / \mathrm{kg}$ did not show a greater effect than $20 \mathrm{mg} / \mathrm{kg}$. The above immunostaining results indicate that $\mathrm{Rb} 1$ protects neurons from LPS-induced damage.

\subsection{Rb1 suppresses LPS-induced microglial activation and IL-1 $\beta$ production in the brain}

Excessively activated microglia are suggested to be directly or indirectly involved in neuroinflammation and consequent neuronal injury $[11,14]$. To understand the neuroprotective effects of $\mathrm{Rb} 1$ following LPS insult, we investigated microglial activation as depicted by IBa-1-positive cells in CA1, S1Tr, S1BF and S2 areas. The production of IL- $1 \beta$, one of the most important pro-inflammatory cytokines released by microglia [23], was also monitored. As shown in Fig. 5, LPS induced an obvious microglial activation with thicker protrusions and larger cell soma (Fig. 5A). The IL- $1 \beta$ in the brain of LPS mice was significantly higher than that in control mice (Fig. 5C). Rb1 treatment significantly suppressed the microglial activation in CA1, S1Tr, S1BF, and S2 areas and inhibited the IL- $1 \beta$ production induced with LPS (Fig. 5A$\mathrm{C})$. The results suggest that $\mathrm{Rb} 1$ effectively attenuated the LPS-induced microglial activation and IL- $1 \beta$ production.

\subsection{Rb1 upregulated the expression of GLT-1 and GS in the brain of LPS mice}

Astrocytes and microglia are two interrelated and interacting cells involved in maintaining brain function. Systemic exposure to endotoxin has been shown to activate microglia and astrocytes [24]. LPS-induced astrocytes activated to A1 subtypes are suggested to be harmful to neurons in the brain [25]. Although the mechanisms by which A1 subtypes act as "bad players" remain unknown, it is helpful to monitor the GLT-1 and GS after LPS insult because more than $90 \%$ of the glutamate released into the synaptic cleft is not taken by GLT- 1 on the astrocyte membrane, and most of the glutamate entering into astrocytes is converted into glutamine by GS to avoid excitotoxicity [26]. As shown in Fig. 6, systemically LPS administration led to reactive astrogliosis in hippocampus CA1 characterized by hypertrophy and an increasing number of GFAP-labeled cells, along with considerable expression of GLT-1 transporter (Fig. 6A,B,E). However, the expression of GS was reduced after LPS treatment (Fig. 6C,D,F). Rb1 $20 \mathrm{mg} / \mathrm{kg}$ and $40 \mathrm{mg} / \mathrm{kg}$ treatments further enhanced the production GLT-1 in astrocytes after LPS insult and significantly attenuated the GS reduction induced by LPS, which was confirmed by both immunostaining and Western Blot analysis. The results reveal that $\mathrm{Rb} 1$ can further increase the expression of GLT-1 and attenuate the GS reduction induced by LPS insult. 
A

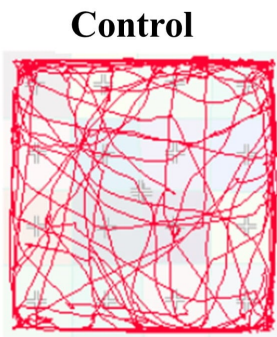

B

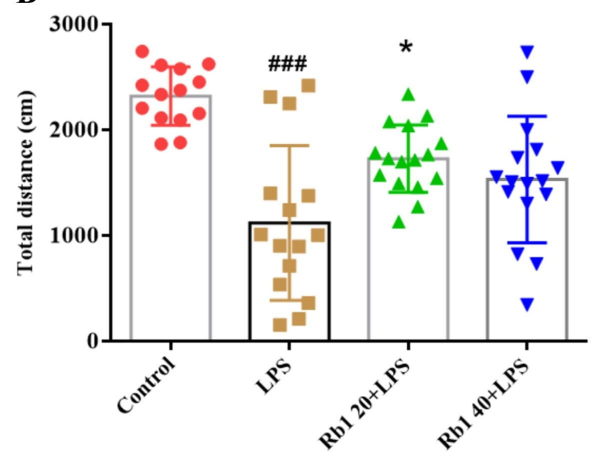

D

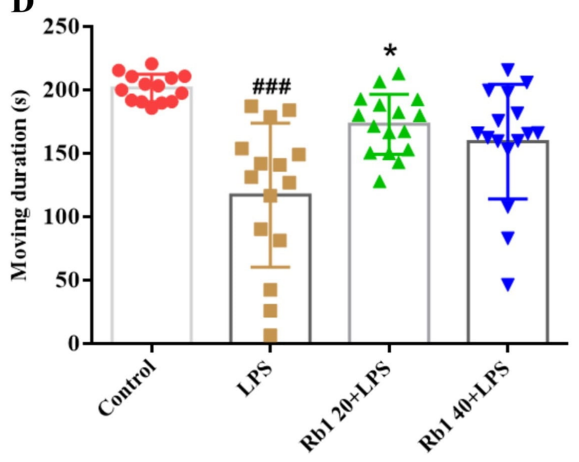

F

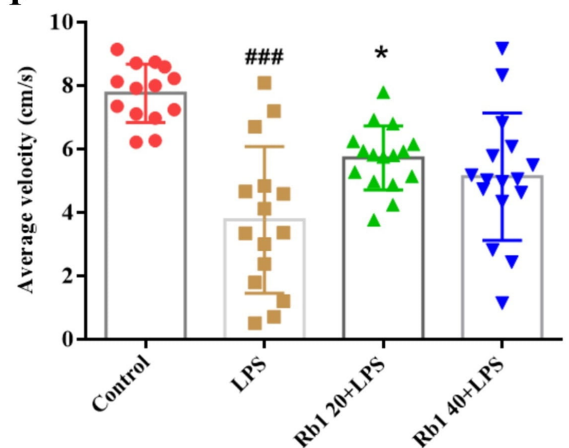

Rb1 20+LPS
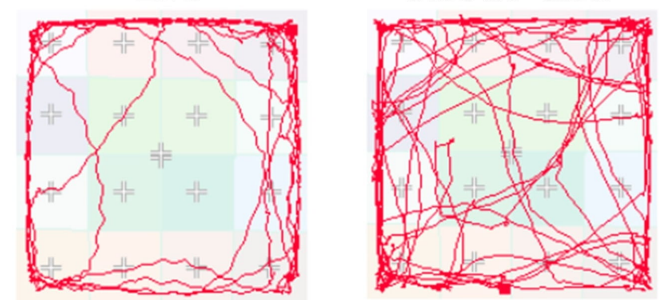

Rb1 40+LPS
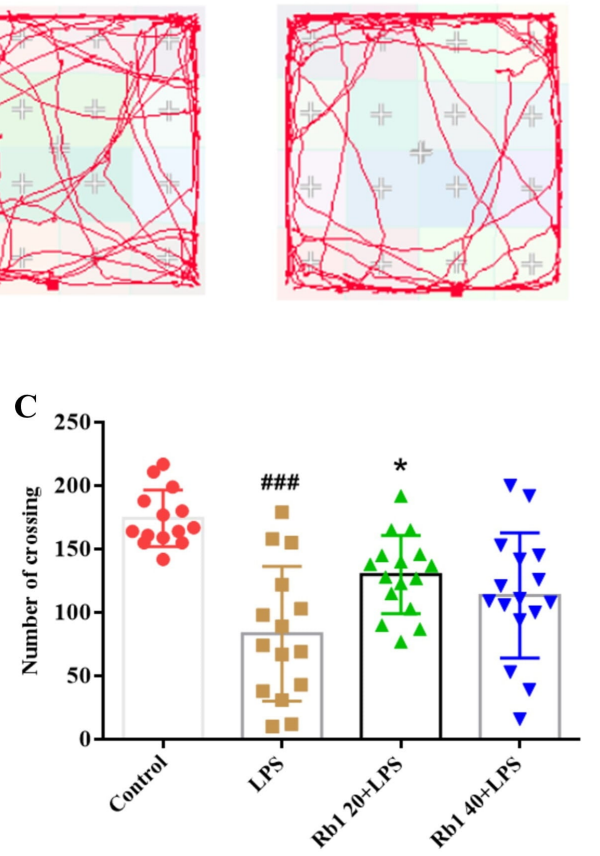

E

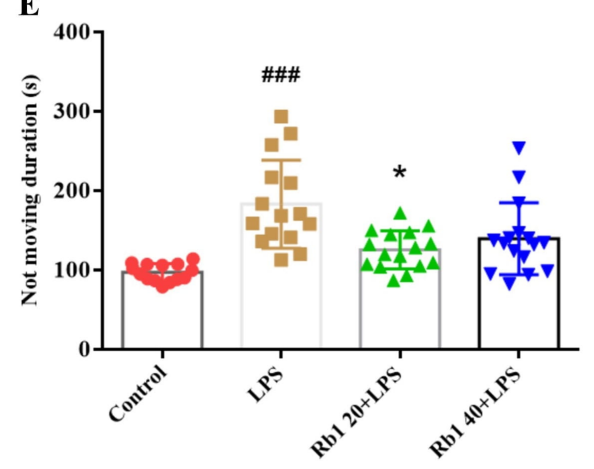

Fig. 2. Rb1 improved the locomotive activities in LPS-treated mice. The locomotive activity was monitored using the open-field test between $20 \mathrm{~h}$ and $24 \mathrm{~h}$ after the LPS injection. The track maps of different groups (A), and quantitative analysis of total distance, crossing lines, moving and stand still duration, average velocity during $5 \mathrm{~min}(\mathrm{~B}-\mathrm{F})$ are listed. Data are represented as mean $\pm \mathrm{SD}(\mathrm{n}=20)$. \#\#\#p<0.001 versus the control group; ${ }^{*} p<0.05$ versus the LPS group.

\section{Discussion}

We demonstrated that Rb1 administered in advance has neuroprotective effects in LPS-injected mice. Rb1 can im- prove the locomotor function and motor coordination of LPS mice. Rb1 can prevent neuronal cell loss and inhibit neuroinflammation in the hippocampus and somatosensory 

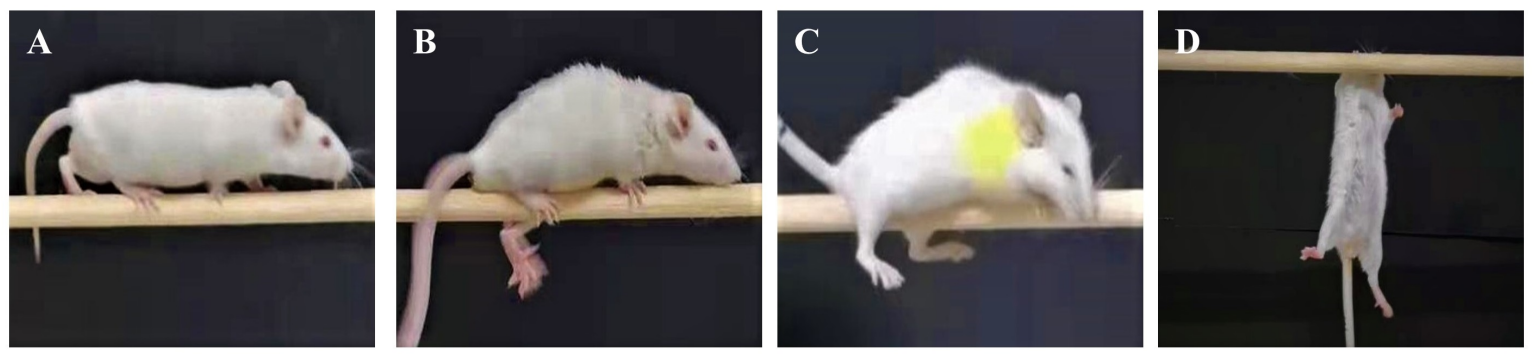

E

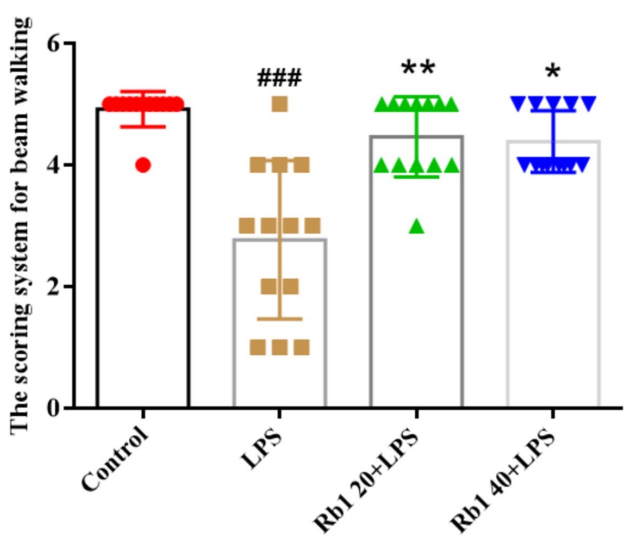

F

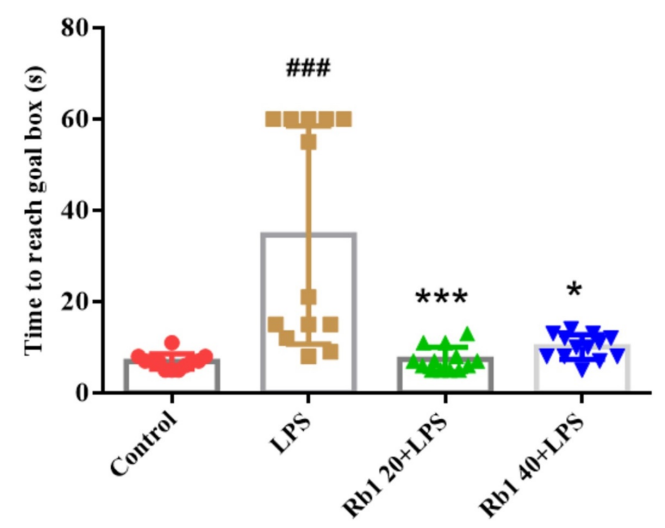

Fig. 3. Rb1 improved LPS-induced motor coordination deficits. The beam walking assay was used to determine motor coordination between $20 \mathrm{~h}$ and 24 $\mathrm{h}$ after LPS injection. The images illustrate the performance for the beam walking test, including traversing the beam normally (A), or hindfoot slips (B) with difficulties to place the hindfoot on the beam (C) or falls from the beam (D). The performance of the mice crossing the beam was as evaluated with scoring (E), and a higher score represents a better performance on the beam. The time crossing the beam to reach the goal box was also recorded (F). Data are represented as mean $\pm \mathrm{SD}(\mathrm{n}=20)$. \#\#\#p<0.001 versus the control group; ${ }^{*} p<0.05,{ }^{* *} p<0.01,{ }^{* * *} p<0.001$ versus the LPS group.

cortex caused by LPS injection. In addition, Rb1 can further enhance the LPS-induced GLT-1 expression and alleviate LPS-induced GS reduction in the brain. Taken together, $\mathrm{Rb} 1$ has neuroprotective effects against LPS-induced neuroinflammatory lesions in the brain of mice. $\mathrm{Rb} 1$ regulation of the expression of GLT-1 and GS may be involved in its effects.

We presumed that the Rb1 improvement in LPS-induced locomotor and motor coordination deficits reduced voluntary activities, decreased locomotor speed, and impaired motor coordination) due to its neuronal protection on the somatosensory cortex and hippocampus. As illustrated by NeuN immunostaining, $24 \mathrm{~h}$ following i.p. injection of LPS at $3 \mathrm{mg} / \mathrm{kg}$, there is evidence of neuronal loss in S1Tr, S1BF, and S2 of the somatosensory cortex, as well as in CA1 of the hippocampus. Rb1 administered on 7 consecutive days in advance significantly alleviated the LPS induced neuronal loss and improved the motor functions in LPS mice. These results not only confirm our presumption as mentioned above, but they also shed light on the findings that Rb1 improves learning and memory, as well as the sensorimotor system, in several other studies [27-29].
In the brain, inflammatory response, termed neuroinflammation, is a fundamental response generated to protect the neuronal system. Microglia is the key player in neuroinflammation in the brain. Microglia engage in environmental surveillance in the "rest" stage by constantly sampling areas around them to maintain homeostasis $[11,30]$. Upon activation, microglia can release several cytokines and process the intruders or "dying" cells to prevent further injury. However, uncontrolled or prolonged microglial activation is potentially harmful and can result in other neuronal lesions. We found that LPS induced a profound microglial activation with significantly increased IL- $1 \beta$ production, which is an indicator of "bad" microglia as M1 stage. Treatment with Rb1 markedly reduced the microglia activation and IL- $1 \beta$ production caused by LPS insult, suggesting an important role of $\mathrm{Rb} 1$ attenuating neuroinflammation in its neuroprotective effects.

Astrocytes play an essential role in maintaining glutamate homeostasis depending on excitatory amino acid transporters (EAATs) and GS. GLT-1 is preferentially expressed in astrocytes and transfers glutamate from the synaptic cleft to astrocytic intracellular space to avoid glutamate excitotoxicity 

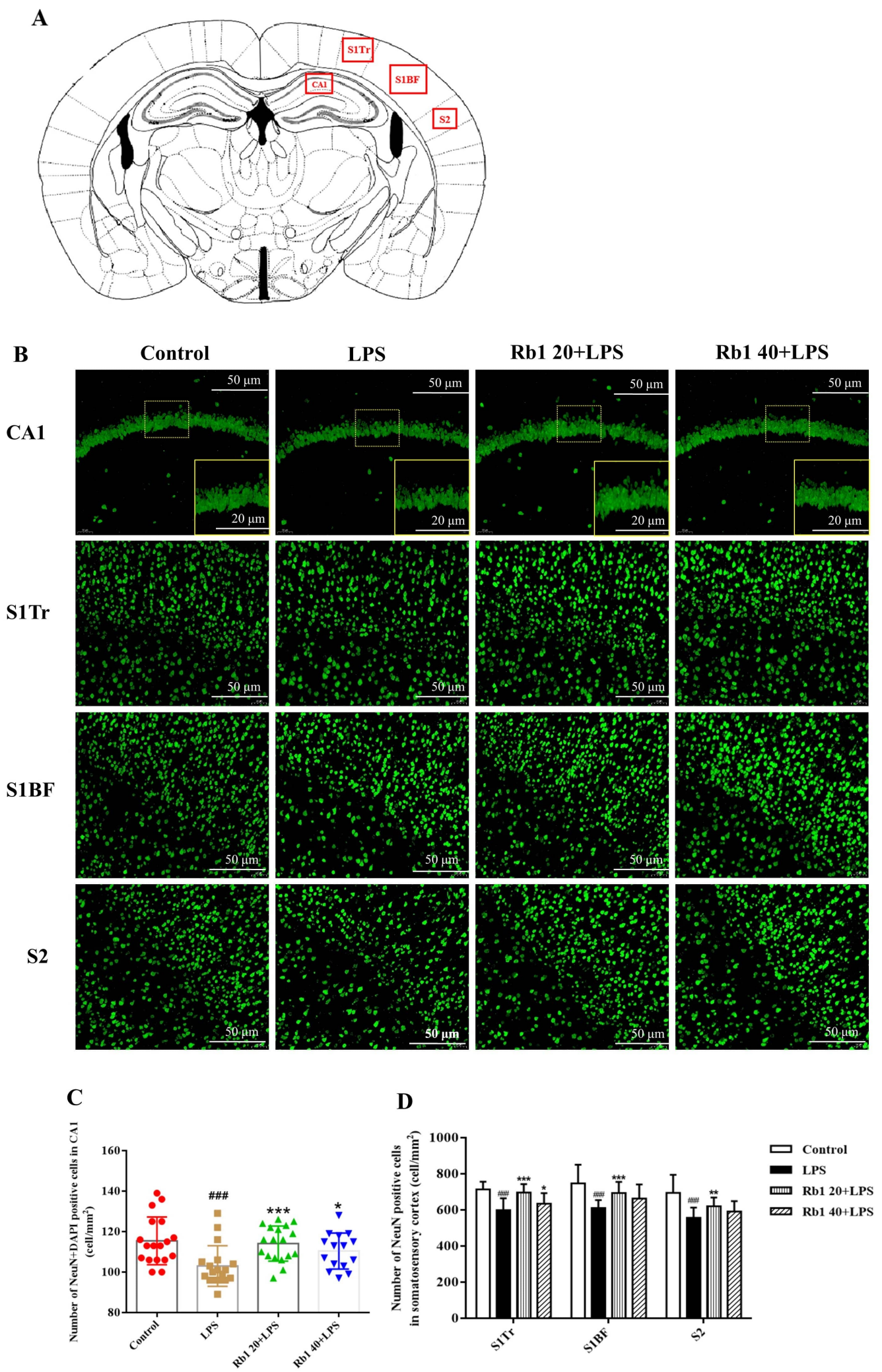

Fig. 4. Rb1 attenuated neuron loss in the brain following LPS treatment. Immunostaining was performed $24 \mathrm{~h}$ after LPS i.p. injection. The NeuN positive cells in CA1, S1Tr, S1BF, and S2 were monitored (Red boxes) (A). The immunostaining of NeuN-labeled cells (Green) was presented in CA1, S1Tr, $\mathrm{S} 1 \mathrm{BF}$, and S2 areas (B). NeuN+DAPI or NeuN positive cells in the hippocampus (C) and somatosensory motor cortex (D) were counted. Data are represented as mean $\pm \mathrm{SD}(\mathrm{n}=6)$. \#\#\# $p<0.001$ versus the control group; ${ }^{*} p<0.05{ }^{* *} p<0.01$, and ${ }^{* * *} p<0.001$ versus the LPS group. 

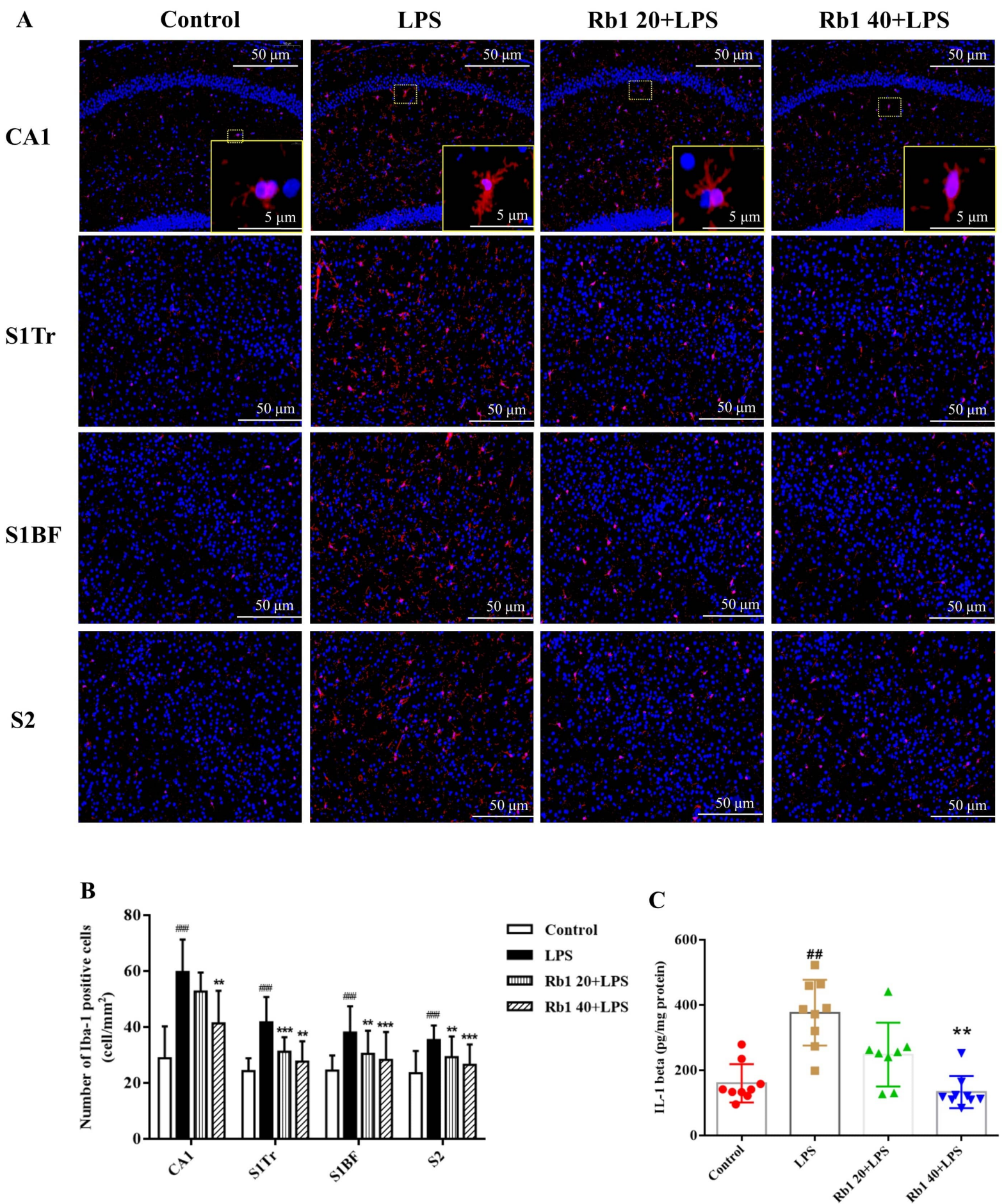

Fig. 5. Rb1 treatment suppressed the LPS-induced microglial activation and inhibited the production of IL- $1 \beta$ in the brain. IBa- 1 immunostaining and the IL-1 $\beta$ test were performed $24 \mathrm{~h}$ after LPS i.p. injection. The IBa-1 positive cells (Red) were shown in CA1, S1Tr, S1BF, and S2 area (A). The nuclei were stained with DAPI (Blue). The IBA1 cells in CA1, S1Tr, S1BF, and S2 were counted (B). The IL- $1 \beta$ production in the brain was analyzed with ELISA (C). Data are represented as mean $\pm \mathrm{SD}(\mathrm{n}=6$ or 9$)$. \#\#\# $p<0.001$ versus the control group; ${ }^{* *} p<0.01$ and ${ }^{* * *} p<0.001$ versus the LPS group.

[31]. Once taken up by astrocytes, in addition to re-entry into the extracellular space or TCA cycle, most of the glutamate coverts to glutamine by GS [26], which serves as the substrate for the synthesis of glutamate and GABA in corresponding neuronal cells. Recent findings suggest that sustained microglial activation leads to failure in astrocytic normal resolu- 

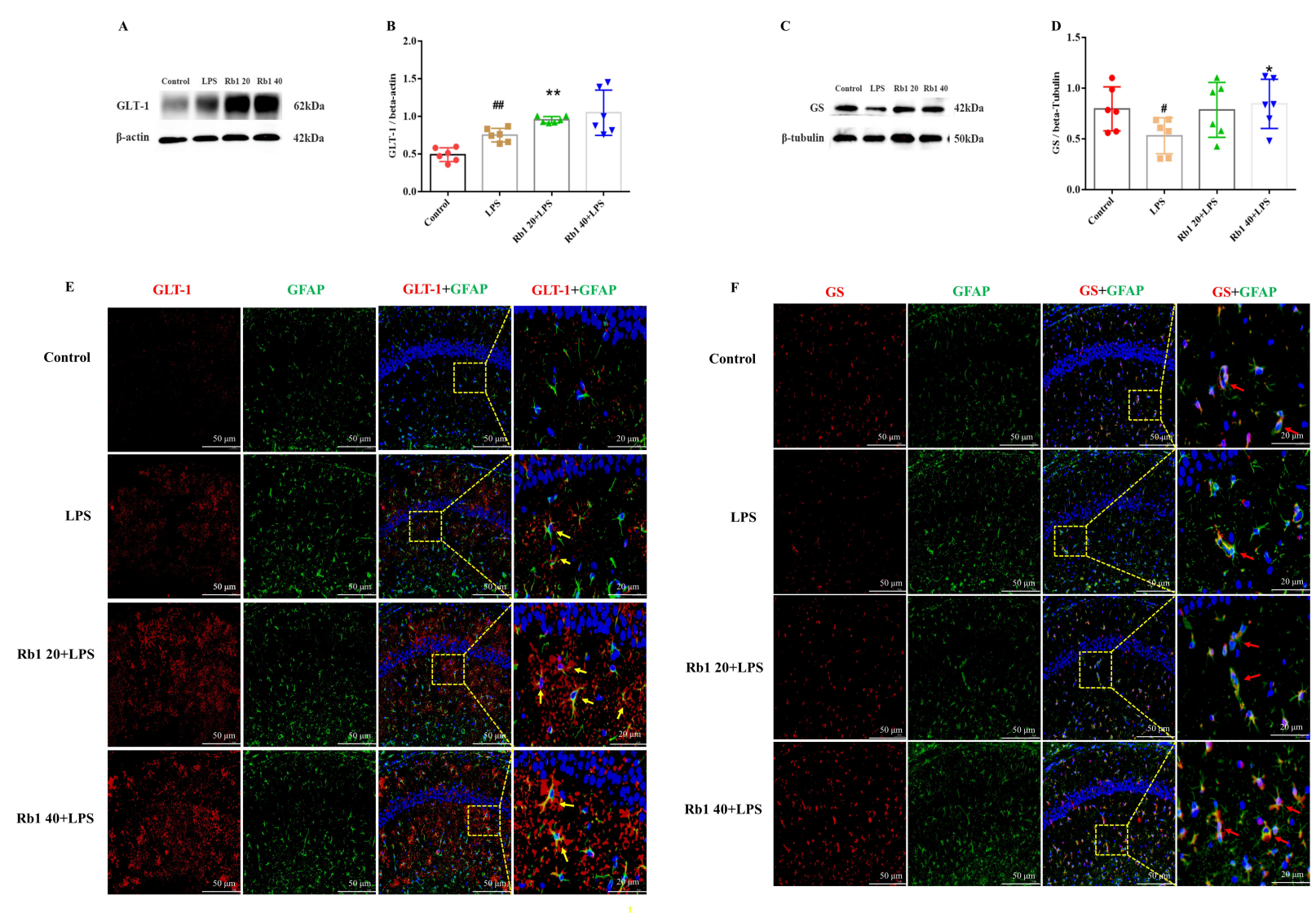

Fig. 6. Rb1 upregulated the expression of GLT-1 and GS in LPS mice. Western Blot and immunostaining were performed 24 h after LPS i.p. injection. Expressions of GLT-1 and GS in the brain were evaluated by Western blot analysis (A,C). The quantification of GLT-1 and GS were normalized by the expression of $\beta$-actin (B,D). Dual immunofluorescence staining with GFAP and GLT-1 or GS in the CA1 region of the hippocampus (E,F). GLT-1 and GS (Red), GFAP (Green). GLT-1 was expressed in astrocytes (Yellow arrows), GS was expressed in astrocytes (Red arrows). Data are represented as mean \pm SD (n = 3 6). \#p $<0.05$, \#\#p $<0.05$ versus the control group; ${ }^{*} p<0.05,{ }^{* *} p<0.01$ versus the LPS group.

tion mechanisms of excitatory glutamate [14, 32,33]. Present findings show that LPS insult resulted in an increased GLT1expression and decreased GS expression, indicating a compromised function of glutamate cycling, which is believed to participate in gliotoxic effects of LPS due to overloading of glutamate inside the astrocytes [32, 34, 35]. Promisingly, for the first time, we found that Rb1 further enhanced the GLT1 expression and significantly increased the GS expression in the brain of LPS mice. This result explains from another perspective why Rb1 has neuroprotective effects against LPS insult.

The ginsenosides, also known as steroid-like saponins, are the major active pharmacological components of ginseng. More than 100 ginsenosides have been identified [36]. Several recent studies have presented evidence showing that ginsenosides could be used to prevent and treat various inflammatory diseases [36]. It was reported that ginsenoside $\mathrm{Rb} 1$ is regarded as the main compound responsible for the neuroprotective effects of ginsenosides [13]. In addition, our previous findings suggest that $\mathrm{Rb} 1$ plays a protective role in astrocytic dysfunction in light of enhanced expres- sion of GLT-1and GS following oxygen and glucose deprivation (OGD/R). We demonstrated that $\mathrm{Rb} 1$ has neuroprotective effects against LPS insult and can regulate the glutamate cycling system, indicated by GLT-1 and GS. Given the importance of glutamate in brain function, it is reasonable to assume that regulating GLT-1/GS may be the common mechanism for $\mathrm{Rb} 1$ protecting neuron lesions in various insults. This is further supported because, in addition to LPS insult and OGD/R insult, we also found that Rb1 can significantly alleviate pentylenetetrazol-induced seizure symptoms in mice and improve GLT-1/GS expression in the brain (Unpublished results).

\section{Conclusions}

In conclusion, our findings indicate that $\mathrm{Rb} 1$ has neuroprotective effects against LPS insults in mice, closely correlated with the Rb1 improvement of somatosensory motor impairment. Rb1 regulation of perturbation glial cells, including microglia and astrocyte, may involve its neuroprotection. Until now, scientific evidence has been lacking as to why ginsenosides (including Rb1) have been reported to 
have neuronal protective effects against several distinct insults. The present work may shed light on understanding the underlying mechanisms. This further understanding of the mechanism by which $\mathrm{Rb} 1$ regulates the GLT-1/GS system further supports its potential for treating neurodegenerative diseases.

\section{Abbreviations}

$\mathrm{BBB}$, blood-brain barrier; CNS, central nervous system; EAATs, excitatory amino acid transporters; Glu, glutamate; Gln, glutamine; GLT-1, glutamate transporter-1; GS, glutamine synthase; GFAP, glial fibrillary acidic protein; IBa-1, ionized calcium-binding adapter molecule-1; IL-1 $\beta$, interleukin- $1 \beta$; OGD/R, oxygen-glucose deprivation/reoxygenation.

\section{Author contributions}

$\mathrm{HZ}$ and MT designed the experiments, HZ, XC, XW, and YL performed and analyzed the data. MT contributed reagents and materials. $\mathrm{HZ}$ wrote the paper, and MT, CDS edited the paper. All authors read and approved the final manuscript.

\section{Ethics approval and consent to participate}

Animals were raised and handled at the Laboratory Animal Center of the Beijing University of Chinese Medicine, Beijing. All animal experiments were carried out in accordance with the National Institutes of Health Guide for Care and Use of Laboratory Animals, and the Institutional Animal Care and Use Committee of Beijing University of Chinese Medicine approved the protocol (BUCM-4-20201008034136, 8 October 2020). The datasets were obtained with the informed consent of all participants.

\section{Acknowledgment}

We thank four anonymous reviewers for their excellent criticism of the article.

\section{Funding}

This research was supported by the grant "International Cooperation Project of Prevention and Treatment of Major Diseases with Chinese Medicine (0610-2040N F020928)", "High-end Experts Support Program from the Ministry of Science and Technology (DL 20200001008)", "Basic Research Funds from the Ministry of Education (No. 1000061223731)”.

\section{Conflict of interest}

The authors declare no conflict of interest.

\section{References}

[1] Ransohoff RM. How neuroinflammation contributes to neurodegeneration. Science. 2016; 353: 777-783.

[2] Kwon HS, Koh S. Neuroinflammation in neurodegenerative disorders: the roles of microglia and astrocytes. Translational Neurodegeneration. 2020; 9: 42 .
[3] Qin L, Wu X, Block ML, Liu Y, Breese GR, Hong J, et al. Systemic LPS causes chronic neuroinflammation and progressive neurodegeneration. Glia. 2007; 55: 453-462.

[4] Erickson MA, Banks WA. Cytokine and chemokine responses in serum and brain after single and repeated injections of lipopolysaccharide: multiplex quantification with path analysis. Brain, Behavior, and Immunity. 2011; 25: 1637-1648.

[5] Logsdon AF, Erickson MA, Chen X, Qiu J, Lim Y, Stonestreet BS, et al. Inter-alpha inhibitor proteins attenuate lipopolysaccharideinduced blood-brain barrier disruption and downregulate circulating interleukin 6 in mice. Journal of Cerebral Blood Flow \& Metabolism. 2020; 40: 1090-1102.

[6] Lee JW, Lee YK, Yuk DY, Choi DY, Ban SB, Oh KW, et al. Neuroinflammation induced by lipopolysaccharide causes cognitive impairment through enhancement of beta-amyloid generation. Journal of Neuroinflammation. 2008; 5: 37.

[7] Hsing C, Hung S, Chen Y, Wei T, Sun D, Wang J, et al. Histone deacetylase inhibitor trichostatin a ameliorated endotoxininduced neuroinflammation and cognitive dysfunction. Mediators of Inflammation. 2015; 2015: 163140.

[8] Li M, Li C, Yu H, Cai X, Shen X, Sun X, et al. Lentivirusmediated interleukin-1 $\beta$ (IL-1 $\beta$ ) knock-down in the hippocampus alleviates lipopolysaccharide (LPS)-induced memory deficits and anxiety- and depression-like behaviors in mice. Journal of Neuroinflammation. 2017; 14: 190.

[9] Daneman R, Prat A. The blood-brain barrier. Cold Spring Harbor Perspectives in Biology. 2015; 7: a020412.

[10] Oberheim NA, Goldman SA, Nedergaard M. Heterogeneity of astrocytic form and function. Methods in Molecular Biology. 2012; 814: 23-45.

[11] Hickman S, Izzy S, Sen P, Morsett L, El Khoury J. Microglia in neurodegeneration. Nature Neuroscience. 2018; 21: 1359-1369.

[12] Ke L, Guo W, Xu J, Zhang G, Wang W, Huang W. Ginsenoside $\mathrm{Rb} 1$ attenuates activated microglia-induced neuronal damage. Neural Regeneration Research. 2014; 9: 252-259.

[13] Cheng Z, Zhang M, Ling C, Zhu Y, Ren H, Hong C, et al. Neuroprotective effects of ginsenosides against cerebral ischemia. Molecules. 2019; 24: 1102.

[14] Tilleux S, Hermans E. Neuroinflammation and regulation of glial glutamate uptake in neurological disorders. Journal of Neuroscience Research. 2007; 85: 2059-2070.

[15] National Research Council. Guide for the care and use of laboratory animals. 8th edn. Washington (DC): National Academies Press. 2011.

[16] Carter RJ, Morton J, Dunnett SB. Motor coordination and balance in rodents. Current Protocols in Neuroscience. 2008; Chapter 8: Unit 8.12.

[17] Stanley JL, Lincoln RJ, Brown TA, McDonald LM, Dawson GR, Reynolds DS. The mouse beam walking assay offers improved sensitivity over the mouse rotarod in determining motor coordination deficits induced by benzodiazepines. Journal of Psychopharmacology. 2005; 19: 221-227.

[18] Quinn LP, Perren MJ, Brackenborough KT, Woodhams PL, Vidgeon-Hart M, Chapman $\mathrm{H}$, et al. A beam-walking apparatus to assess behavioural impairments in MPTP-treated mice: pharmacological validation with R-(-)-deprenyl. Journal of Neuroscience Methods. 2007; 164: 43-49.

[19] Kraeuter A, Guest PC, Sarnyai Z. The open field test for measuring locomotor activity and anxiety-like behavior. Methods in Molecular Biology. 2019; 1916: 99-103.

[20] Feeney DM, Gonzalez A, Law WA. Amphetamine, haloperidol, and experience interact to affect rate of recovery after motor cortex injury. Science. 1982; 217: 855-857.

[21] Karadimas SK, Satkunendrarajah K, Laliberte AM, Ringuette D, Weisspapir I, Li L, et al. Sensory cortical control of movement. Nature Neuroscience. 2020; 23: 75-84.

[22] Berdasco C, Pinto A, Calabró V, Arenas D, Cangelosi A, Geoghegan $\mathrm{P}$, et al. Shiga toxin 2 from enterohemorrhagic Escherichia coli induces reactive glial cells and neurovascular disarrangements 
including edema and lipid peroxidation in the murine brain hippocampus. Journal of Biomedical Science. 2019; 26: 16.

[23] Spulber S, Schultzberg M. Connection between inflammatory processes and transmittor function-modulatory effects of interleukin-1. Progress in Neurobiology. 2010; 90: 256-262.

[24] Okuyama S, Makihata N, Yoshimura M, Amakura Y, Yoshida $\mathrm{T}$, Nakajima M, et al. Oenothein B suppresses lipopolysaccharide (LPS)-induced inflammation in the mouse brain. International Journal of Molecular Sciences. 2013; 14: 9767-9778.

[25] Liddelow SA, Guttenplan KA, Clarke LE, Bennett FC, Bohlen CJ, Schirmer L, et al. Neurotoxic reactive astrocytes are induced by activated microglia. Nature. 2017; 541: 481-487.

[26] Coulter DA, Eid T. Astrocytic regulation of glutamate homeostasis in epilepsy. Glia. 2012; 60: 1215-1226.

[27] Wang Q, Sun L, Jia W, Liu X, Dang H, Mai W, et al. Comparison of ginsenosides Rg1 and Rb1 for their effects on improving scopolamine-induced learning and memory impairment in mice. Phytotherapy Research. 2010; 24: 1748-1754.

[28] Shi YH, Li Y, Wang Y, Xu Z, Fu H, Zheng GQ. Ginsenoside-Rb1 for ischemic stroke: a systematic review and meta-analysis of preclinical evidence and possible mechanisms. Frontiers in Pharmacology. 2020; 11: 285.

[29] Yang Y, Li S, Huang H, Lv J, Chen S, Pires Dias AC, et al. Comparison of the protective effects of ginsenosides $\mathrm{rb} 1$ and rg1 on improving cognitive deficits in SAMP8 mice based on anti-neuroinflammation mechanism. Frontiers in Pharmacology. 2020; 11: 834
[30] Glass CK, Saijo K, Winner B, Marchetto MC, Gage FH. Mechanisms underlying inflammation in neurodegeneration. Cell. 2010; 140: 918-934.

[31] Garcia-Esparcia P, Diaz-Lucena D, Ainciburu M, TorrejónEscribano B, Carmona M, Llorens F, et al. Glutamate transporter GLT1 expression in Alzheimer disease and dementia with Lewy bodies. Frontiers in Aging Neuroscience. 2018; 10: 122.

[32] Tilleux S, Goursaud S, Hermans E. Selective up-regulation of GLT-1 in cultured astrocytes exposed to soluble mediators released by activated microglia. Neurochemistry International. 2009; 55: 35-40.

[33] Takaki J, Fujimori K, Miura M, Suzuki T, Sekino Y, Sato K. Lglutamate released from activated microglia downregulates astrocytic L-glutamate transporter expression in neuroinflammation: the 'collusion' hypothesis for increased extracellular L-glutamate concentration in neuroinflammation. Journal of Neuroinflammation. 2012; 9: 275.

[34] Chen CJ, Liao SL, Kuo JS. Gliotoxic action of glutamate on cultured astrocytes. Journal of Neurochemistry. 2000; 75: 1557-1565

[35] Sharma A, Patro N, Patro IK. Lipopolysaccharide-induced apoptosis of astrocytes: therapeutic intervention by minocycline. Cellular and Molecular Neurobiology. 2016; 36: 577-592.

[36] Kim JH, Yi Y, Kim M, Cho JY. Role of ginsenosides, the main active components of Panax ginseng, in inflammatory responses and diseases. Journal of Ginseng Research. 2017; 41: 435-443. 